\title{
Posterior reconstruction and anterior suspension with single anastomotic suture in robot-assisted laparoscopic radical prostatectomy: a simple method to improve early return of continence
}

\author{
Jonathan F. Kalisvaart • Kathryn E. Osann • \\ David S. Finley • David K. Ornstein
}

Received: 9 June 2009 / Accepted: 12 July 2009 / Published online: 6 August 2009

(c) The Author(s) 2009. This article is published with open access at Springerlink.com

\begin{abstract}
Post-prostatectomy urinary incontinence is a major cause of morbidity from radical prostatectomy. Efforts have been made to develop techniques to hasten return of urinary control. Several authors have demonstrated improved early continence with anterior, posterior, or combined reconstruction of the urethral-pelvic attachments. In this study, we compare three-month urinary function and continence data for patients who underwent RALP with posterior reconstruction and anterior suspension with single anastomotic suture (PRASS). A prospective cohort of 50 patients underwent RALP with PRASS reconstruction and were compared to 50 control patients who underwent standard RALP. Continence was defined as use of $0-1$ urinary pads and was evaluated at each follow-up visit using the EPIC-26 questionnaire. A weighted summary score was created and group differences were compared using a repeated measures analysis of variance model. After adjusting for age, baseline AUA symptom score, and SHIM scores, which were found to correlate with continence, patients who underwent the PRASS reconstruction had significantly improved urinary control at three months compared with the control group; $90.9 \%$ of the patients in the
\end{abstract}

\footnotetext{
J. F. Kalisvaart · D. S. Finley · D. K. Ornstein Department of Urology, University of California, Irvine Medical Center, Orange, CA 92868, USA e-mail: jonkalisvaart@gmail.com

K. E. Osann

Department of Medicine, University of California, Irvine Medical Center, Orange, CA 92868, USA

D. K. Ornstein $(\square)$

Vanguard Urologic Institute, Memorial Hermann Medical Plaza, 6400 Fanin St., Suite 2300, Houston, TX 77030, USA

e-mail: jonkalisvaart@gmail.com
}

PRASS group wore $0-1$ pads per day versus $48.2 \%$ in the control group $(P=0.014)$. Of the patients undergoing the standard prostatectomy $20.6 \%$ were totally pad-free compared with $42 \%$ of the patients undergoing the PRASS procedure $(P=0.042)$. In conclusion, the PRASS technique resulted in statistically significant improvement in urinary control three months post-operation. The PRASS reconstruction is technically straightforward, requires no additional sutures, and is a simple technique that is easily learned and adaptable to other robotic surgery.

Keywords Robotics · Prostatectomy · Urinary incontinence
Abbreviations
BMI Body mass index
EPIC European prospective investigation into cancer and nutrition
RALP Robotic assisted laparoscopic prostatectomy
SHIM Sexual health inventory for men

\section{Introduction}

Short-term urinary incontinence is a major cause of morbidity associated with both the open and standard robotic/ laparoscopic radical prostatectomy. Although fewer than 5$10 \%$ of patients have long-term effects after one year, the incidence of early post-operative incontinence varies widely, with $44-76 \%$ of patients requiring two or more pads per day at three months [1-3]. Despite the transient nature in most cases, quality-of-life studies have revealed that short-term incontinence is severely bothersome to most men [4]. Recent studies have attempted to preserve early continence by anatomically restoring the urethral-vesical 
junction [5-9]. Although early data suggests that some of these techniques may improve early urinary control, some are technically demanding and may prolong operative times for less experienced surgeons. The most effective and efficient method to improve early return of urinary control following RALP remains to be established.

In an effort to improve short-term urinary control we developed a simplified approach to combined posterior reconstruction and anterior suspension using a single anastomotic stitch (PRASS). Herein, we prospectively evaluated recovery of urinary control in a single-surgeon-series of patients undergoing PRASS and compared them with a cohort who underwent standard RALP.

\section{Materials and methods}

Following approval from the UCI Internal Review Board, data were collected prospectively by patient reported selfadministered questionnaires obtained during preoperative and three month post-operative visits following RALP. All cases were performed by a single surgeon (DKO) between November 2003 and May 2008. In the first 231 procedures the urethral-vesical anastomosis was completed using the standard Van Velthovan stitch as previously described [10] without any additional anti-incontinence procedures. In July of 2007, the PRASS modification was introduced. Fifty consecutive patients were evaluated (case \#275-324) and compared with 50 consecutive patients who underwent standard RALP (control group; case \#182-231). Baseline patient characteristics are shown in Table 1. At three months, follow-up data were available for 39 patients (78\%) in the control group and 35 patients $(70 \%)$ in the PRASS group. Follow-up data were not available for 11 control and 15 PRASS patients because these patients had returned to their local urologist for follow-up care. There was no significant difference in baseline characteristics of the patients with and without three month follow-up data.

\section{Statistical analysis}

\section{EPIC summary score}

Baseline patient characteristics (age, AUA symptom score, SHIM score, nerve sparing procedure, BMI, prostate weight, positive surgical margin rate) were analyzed to assess for preoperative differences between groups (Table 1). Continence was defined as using $0-1$ pads per day. Post-operative continence was evaluated by using questions 1-5 from the EPIC-26 questionnaire:

1 "Over the past 4 weeks how often have you leaked urine?"
Table 1 Baseline characteristic of (a) patients with and without three months follow-up data and (b) patients in the control and PRASS groups

\begin{tabular}{|c|c|c|c|c|c|}
\hline & \multicolumn{2}{|c|}{$\begin{array}{l}\text { Patient with } \\
3 \text { month } \\
\text { follow-up data }\end{array}$} & \multicolumn{2}{|c|}{$\begin{array}{l}\text { Patients without } \\
3 \text { month } \\
\text { follow-up data }\end{array}$} & \multirow[t]{2}{*}{$P$-Value } \\
\hline & $N$ & Mean & $N$ & Mean & \\
\hline (a) Age (years) & 72 & 63.5 & 28 & 62.3 & 0.45 \\
\hline BMI & 66 & 28.2 & 27 & 28.4 & 0.79 \\
\hline Prostate volume (cc) & 58 & 52.9 & 21 & 50.4 & 0.66 \\
\hline SHIM-pre-surgery & 71 & 19.7 & 28 & 18.6 & 0.64 \\
\hline AUA SS—pre-surgery & 71 & 9.7 & 28 & 11.6 & 0.32 \\
\hline \multirow[t]{2}{*}{ EPIC_-sum at baseline } & 72 & 7.6 & 24 & 6.9 & 0.36 \\
\hline & & Control & & PRASS & $P$ value \\
\hline (b) Age (years) & & 63.8 & & 62.6 & 0.41 \\
\hline BMI & & 29.8 & & 28.6 & 0.53 \\
\hline Prostate volume (cc) & & 53.0 & & 51.4 & 0.75 \\
\hline Pre-surgery SHIM & & 20.5 & & 18.3 & 0.32 \\
\hline Pre-surgery AUASS & & 10.7 & & 9.8 & 0.57 \\
\hline Pre-surgery EPIC sum & & 7.6 & & 7.3 & 0.64 \\
\hline Nerve sparing $(\%)$ & & 70 & & 70 & 1.0 \\
\hline Positive margins (\%) & & 11.6 & & 12.5 & 0.9 \\
\hline
\end{tabular}

2 "Which of the following best describes your urinary control during the last 4 weeks?"

3 "How many pads or adult diapers per day did you usually use to control leakage?"

4 "How big a problem if any has dripping or leaking urine been for you during the last 4 weeks?"

5 "Overall, how big a problem has urinary function been for you during the last 4 weeks?"

A weighted summary score was created from these five items. All responses were scored from a low of one for a favorable response (e.g., no problem) to a high of four or five for an unfavorable response (e.g., significant problem). Response codes were thus reversed for questions 1 and 2 and modified to range from $1-4$ and $1-5$ for questions 3 and $4 \mathrm{a}$, respectively, rather than $0-3$ or 4 . A weighted sum of all responses was calculated so that the highest score (4 or 5) received equal weight for each question. Summary scores ranged from a low of 5.5 to a high of 25 . Correlations between items were high, ranging from $0.46-0.86$. Cronbach's alpha for the summary score was 0.89 indicating high internal consistency.

Data were compared between groups at baseline and at three month follow-up using two-group $t$-tests for continuous variables and Pearson's chi-squared tests for categorical variables. Unadjusted EPIC summary scores were compared between groups using two-group $t$-tests. To adjust for 
possible baseline differences in individuals and to adjust for co-variables independently associated with return to continence, repeated measures analysis of variance model was used. EPIC summary score at baseline and at three month follow-up were used as the within-group repeated measure. Independent covariates which are constant across trials included age, nerve sparing, baseline AUA symptom scores, and SHIM scores. A significant difference between surgical groups in the time by group interaction reflects a difference in the change in continence over time between the two groups.

Using a repeated measures analysis of variance model, we tested for differences between surgical groups in change in continence from baseline to the three month follow-up time point. EPIC summary score was the within-group repeated measure and surgical procedure was the grouping factor. Age, pre-surgery AUA symptoms scores, and pre-surgery SHIM scores were found to be significantly correlated with continence at three months as assessed using the EPIC summary score. Thus these covariates were included in the final repeated measures model to increase precision and adjust for any initial imbalances between groups related to these factors. Nerve sparing, BMI, and prostate volume were not significantly correlated with continence at three months and were excluded from the multivariate and repeated measures model.

\section{Results}

After adjusting for age and baseline AUA symptom score and SHIM score, subjects who received the PRASS procedure had significantly better urinary control at three months. $90.9 \%$ of patients in the PRASS group required one or fewer pads per day compared with $48.2 \%$ in the control group $(P=0.014$, Table 2$)$. Of patients undergoing RALP with the PRASS procedure $42 \%$ were pad-free at three months compared with only $20.6 \%$ of patients undergoing standard RALP $(P=042)$.

The unadjusted EPIC score at three months was not statistically different between the PRASS and RALP groups ( $P$-value 0.96). However, when adjusted for baseline EPIC score, age, AUA symptom score at baseline, and SHIM at baseline the difference was statistically different ( $P$-value 0.013; Table 2b).

There were no clinically significant urine leaks or early bladder neck contractures in either of the groups. In all patients the Foley catheter was removed without a cystogram on POD\# 7-9. Two patients (4\%) in the PRASS group developed urinary retention that resolved after rein-
Table 2 Multivariate analysis results comparing (a) 0-1 pads per day and 0 pads per day (EPIC Question 3) in PRASS and control (RALP) groups at three months post-prostatectomy after controlling for age, and baseline AUA and SHIM scores, and (b) EPIC sum score results adjusted for baseline EPIC score, age, baseline AUA symptom score, and baseline SHIM score

\begin{tabular}{|c|c|c|c|c|c|}
\hline & \multicolumn{4}{|c|}{ Percent using $0-1$ pads $(\%)$} & $P$-value \\
\hline (a) Control & \multicolumn{4}{|l|}{42.8} & 0.014 \\
\hline PRASS & \multicolumn{4}{|l|}{90.9} & \\
\hline & \multicolumn{4}{|c|}{ Percent using 0 pads (\%) } & \\
\hline Control & \multicolumn{4}{|l|}{20.6} & 0.042 \\
\hline PRASS & \multicolumn{4}{|l|}{42.0} & \\
\hline \multicolumn{2}{|l|}{ Group } & $N$ & Mean & SE & $P$-value \\
\hline \multicolumn{2}{|c|}{ (b) Standard group (control) } & 34 & 16.0 & 0.89 & 0.013 \\
\hline \multicolumn{2}{|l|}{ PRASS } & 37 & 12.8 & 0.85 & \\
\hline
\end{tabular}

sertion of the Foley catheter for one week. No patients in the control group had urinary retention.

\section{Comments}

Urinary incontinence is a major health related quality-of-life concern for patients undergoing open or robotic/laparoscopic radical prostatectomy. Immediately following a radical prostatectomy estimates of patients experiencing incontinence vary widely from 8 to $47 \%$ [2,3]. After approximately $12-18$ months the majority of these patients will have regained urinary continence $[1,11]$. Despite favorable long-term outcomes, the duration until full recovery of continence is a significant concern to patients. Quality-oflife studies suggest that even early transient incontinence itself is more bothersome to patients than impotence [4].

Urodynamic studies have demonstrated post-prostatectomy incontinence to be primarily because of intrinsic sphincteric deficiency [12-16]. Multiple attempts have been made to modify Walsh's anatomic radical prostatectomy to prevent injury to, or to repair, the rhabdo-urinary sphincter. Rocco and coworkers described a technique for reconstruction of the posterior musculofascial plate and recreation of the pre-existing continuity between the Denonvilliers' fascia and the posterior rhabdosphincter. The rationale for this maneuver is to provide support to the urethra and restore it to a more anatomic position [5]. In follow-up, the group undergoing the posterior reconstruction had a significantly higher continence rate, defined as $0-1$ pads per day, compared with a historical group undergoing a standard Walsh prostatectomy at 3 days (62.4 vs. $14 \%$ ), 30 days (74 vs. $30 \%$ ), and 90 days ( 85.2 vs. $46 \%)$, 
$P<0.001$. A follow-up report in 2007 also showed that this technique is feasible by a laparoscopic approach, demonstrating improvements in urinary control at 30 days, with $83.8 \%$ of patients with reconstruction having control versus $32.3 \%$ of those undergoing a standard laparoscopic prostatectomy $(P=0.0001)$ [6]. In 2008, Nguyen et al. obtained similar good results for patients undergoing either laparoscopic or robotic prostatectomy. In this series, urethral length was restored to $89 \%$ of its baseline compared with $78 \%$ without the posterior reconstruction, providing objective data to explain the improvement in continence rates [7]. In 2008 Tewari and coworkers evaluated a group of patients undergoing anterior reconstruction alone versus anterior and posterior reconstruction versus a historical control group and found that both types of reconstruction were significantly better than no reconstruction in terms of time to continence, defined as 0 pads per day or a single pad for security reasons only. At three months, the control group had a continence rate of 50\% whereas the anterior reconstruction group and the combined anterior and posterior reconstruction groups had a continence rate of 77 and $91 \%$, respectively, at three months. A much more extensive reconstruction was used in this study than that described here, with multiple interrupted sutures used to reapproximate the posterior urethral plate and a running suture used to reapproximate the arcus tendineus and puboprostatic plate to the bladder neck [8]. Interestingly, a prospective randomized study by Menon et al. in 2008 found there was no difference in early continence ( $<30$ days) between patients undergoing RALP with periprostatic tissue reconstruction and those undergoing a standard single-layer anastamosis [9].

In this study we compared the continence rates at three months in two groups of patients undergoing RALP-one with a posterior reconstruction and anterior suspension with single anastomotic suture (PRASS) and a cohort without these modifications. Unlike previous studies with anterior and/or posterior repairs requiring extensive reconstruction and multiple sutures, we used a simplified technique using only the anastomotic sutures themselves. The posterior repair was performed by using the initial passes of the Van Velthoven suture consisting of 2 3-0 Monocryl sutures [10] to reapproximate the posterior rhabodosphincter of the urethra (Fig. 1a). Unlike the Rocco or Tewari techniques, an extensive repair was not attempted. The anterior suspension stitch was performed at the end of the urethral-vesical anastomosis. After the anastomosis was completed and tied, one of the two arms of the Van Velthoven suture was used to suspend the urethra from the posterior side of the pubic symphysis. The stitch was secured with a single Lapra-Ty (Ethicon) clip (Fig. 1b).

In our study, multivariate analysis demonstrated that, after adjusting for age and baseline AUA symptoms scores
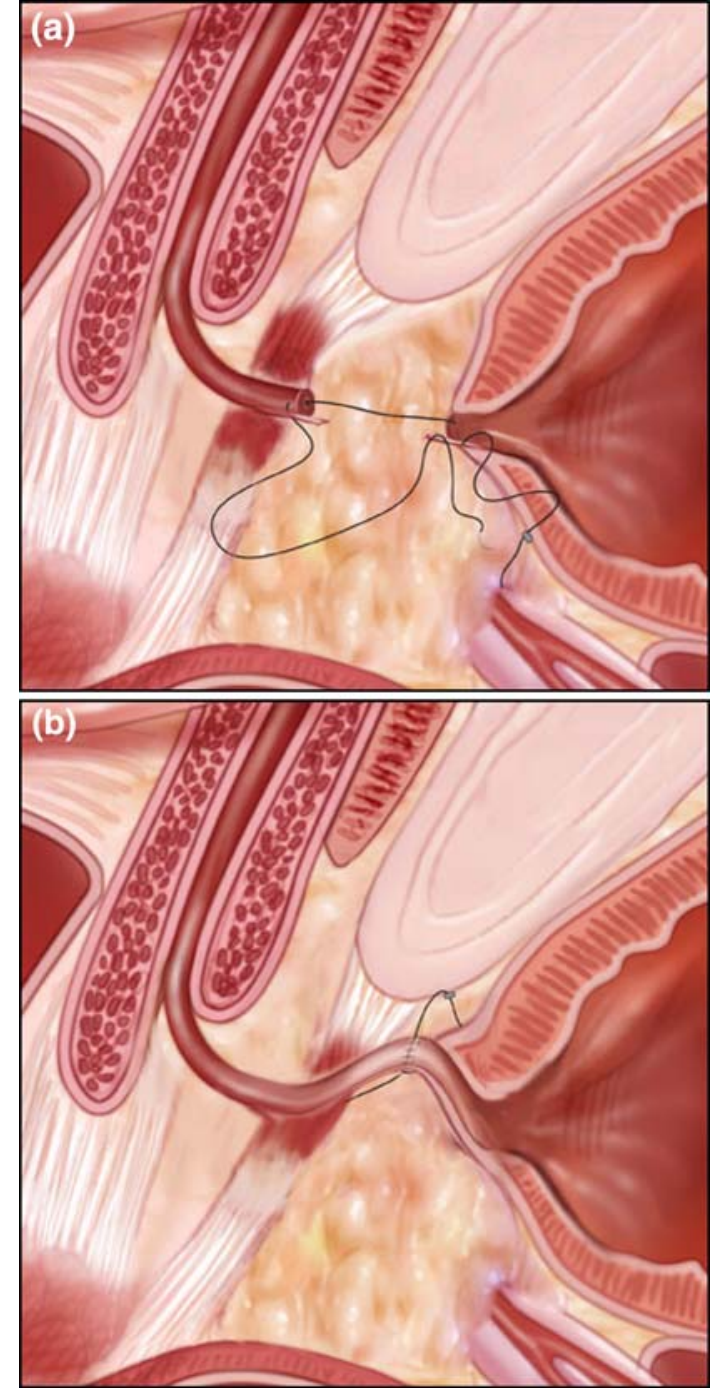

Fig. 1 Illustrations showing the PRASS reconstruction. a The posterior repair is performed by using the initial passes of the Van Velthoven suture to reapproximate the posterior rhabodosphincter of the urethra. b The anterior reconstruction is performed by using one of the two arms of the Van Velthoven suture to suspend the urethra from the posterior side of the pubic symphysis. The stitch was secured with a single Lapra-Ty (Ethicon) clip

and SHIM scores, subjects who underwent PRASS repair had significantly improved urinary control at three months compared with those undergoing a standard RALP. Ninetyone percent $(90.9 \%)$ of the patients undergoing the PRASS repair were using $0-1$ pad per day at three months versus $48.2 \%$ in the control group $(P=0.014)$. This continence rate is comparable with that described by other groups using more extensive reconstructions, both posterior alone [6] and anterior and posterior [8]. The mechanism of improved continence with our technique is restoration of the anatomic configuration of the urethra created with the anterior and posterior reinforcement, as has been successfully done with other, more extensive reconstructions. Our 
technique also does not require multiple interrupted sutures to reconstruct the posterior urethra. This eliminates multiple knots making it simpler and therefore potentially quicker and more available to less experienced surgeons compared with similar, previously described, techniques.

A potential limitation of our study is that it is a case-controlled study rather than a prospective randomized trial. Another, potential limitation is short follow-up of only three months. We believe that three months is a relevant time point and that longer follow-up will not alter the conclusions of this report because, in general, once men regain continence following surgery they do not lose it again. Of course longer term follow-up is needed to determine the true, long term continence rate. In addition, all the men in the PRASS group underwent both an anterior and a posterior repair, making it hard to determine the impact of either the anterior or posterior stitch alone. This is similar to findings in other studies in which reconstruction of any kind was superior to no reconstruction. Men in our study have not undergone urodynamic studies which could be an objective method to measure continence, avoiding the problems inherent to any questionnaire [8]. Last, imaging studies such as MRI could be used to evaluate the impact of our repair on factors such as urethral length.

Despite these potential limitations, this technique does show considerable promise for improving the time to continence in men undergoing robot-assisted laparoscopic prostatectomy. Our technique is simple and easy to perform and has resulted in a significant improvement in continence three months following RALP.

\section{Conclusion}

We have developed a novel, simplified technique for reconstruction of the anterior and posterior support of the urethra to improve time to continence in men undergoing roboticassisted laparoscopic prostatectomy. Patients who had posterior reconstruction and anterior suspension with single anastomotic suture (PRASS) had higher continence rates at three months using this procedure. Further study is needed to determine the different roles of these stitches.

Open Access This article is distributed under the terms of the Creative Commons Attribution Noncommercial License which permits any noncommercial use, distribution, and reproduction in any medium, provided the original author(s) and source are credited.

\section{References}

1. Payromaure M, Ravery V, Boccon-Gibod L (2002) The management of stress urinary incontinence after radical prostatectomy. BJU Int 90:155-161

2. Catalona WJ, Carvalhal GF, Mager DE, Smith DS (1999) Potency, continence and complication rates in 1,870 consecutive radical retropubic prostatectomies. J Urol 162:433-438

3. Fowler FJ Jr, Barry MJ, Lu-Yao G, Roman A, Wasson J, Wennberg JE (1993) Patient-reported complications and follow-up treatment after radical prostatectomy: The national Medicare experience 1988-1990. Urology 42:622-629

4. Litwin MS, Hays RD, Fink A, Ganz PA, Leake B, Leach GE et al (1995) Quality-of-life outcomes in men treated for localized prostate cancer. JAMA 273:129-135

5. Rocco F, Carmignani L, Acquati P, Gadda F, Dell-Orto P et al (2006) Restoration of posterior aspect of rhabdosphincter shortens continence time after radical retropubic prostatectomy. J Urol 175:2201-2206

6. Rocco B, Gregori A, Stener S, Santoro L, Bozzola A et al (2007) Posterior reconstruction of the rhabdosphincter allows a rapid recovery of continence after transperitoneal videolaparoscopic radical prostatectomy. Eur Urol 51:996-1003

7. Nguyen MM, Kamoi K, Stein RJ, Aron M, Hafron JM et al (2008) Early continence outcomes of posterior musculofascial plate reconstruction during robotic and laparoscopic prostatectomy. BJU Int 101:1135-1139

8. Tewari A, Jhaveri J, Rao S, Yadev R, Bartsch G et al (2008) Total reconstruction of the vesico-urethral junction. BJU Int 101:871877

9. Menon M, Muhletaler F, Campos M, Peabody JO (2008) Assessment of early continence after reconstruction of the periprostatic tissues in patients undergoing computer assisted (robotic) prostatectomy: results of a two group parallel randomized controlled trial. J Urol 180:1018-1023

10. Van Velthoven RF, Ahlering TE, Peltier A, Skarecky DW, Clayman RV (2003) Technique for laparoscopic running anastamosis: the single knot method. Urology 61:699-702

11. Stanford JL, Feng Z, Hamiton AS, Gilliland FD, Stephenson RA et al (2000) Urinary and sexual function after radical prostatectomy for clinically localized prostate cancer: The Prostate Cancer Outcomes Study. JAMA 283:354-360

12. Hammerer P, Huland H (1997) Urodynamic evaluation of changes in urinary control after radical retropubic prostatectomy. J Urol 157:233-236

13. Kielb SJ, Clemens JQ (2005) Comprehensive urodynamics evaluation of 146 men with incontinence after radical prostatectomy. Urology 66:392-396

14. Groutz A, Blaivas JG, Chaikin DC, Weiss JP, Verhaaren M (2000) The pathophysiology of post-radical prostatectomy incontinence: a clinical and video urodynamic study. J Urol 163:1767-1770

15. Ficazzola MA, Nitti VW (1998) The etiology of post-radical prostatectomy incontinence and correlation of symptoms with urodynamic findings. J Urol 160:1317-1320

16. Burnett AL, Mostwin JL (1998) In situ anatomical study of the male urethral sphincteric complex: relevance to continence preservation following major pelvic surgery. J Urol 160:1301-1306 